\title{
A Case of Intracranial Hemorrhage in a Neonate with Osteogenesis Imperfecta Type III with a Novel Mutation in COL $1 A 2$
}

\section{Shin-Hee Kim ${ }^{1}$ and Won-Ho Hahn ${ }^{2 *}$ \\ ${ }^{1}$ Department of Pediatrics, College of Medicine, Catholic University, Republic of Korea \\ ${ }^{2}$ Department of Pediatrics, College of Medicine, Soon Chun Hyang University, Republic of Korea}

\begin{abstract}
Background: Osteogenesis imperfecta (OI) is a heritable bone disorder characterized by fractures with minimal trauma. Despite the detailed information about skeletal abnormalities, data about the involvement of neurovascular structures in osteogenesis imperfecta (OI) is limited.

Methods: We report a case of male neonate with OI type III who presented with intracranial hemorrhage. OI type III was diagnosed on the basis of family history and clinical, radiologic and genetic test findings. Results: A novel genetic mutation was found; splicing mutation heterozygous c.1036-2A $>$ C (IVS19) in COL1A2 gene.

Conclusion: This case demonstrates that intracranial hemorrhage could be a major complication in patients with OI type III by underlying unknown mechanisms. OI seems to be an entity associated with intracranial hemorrhage. Thus, we suggest a need for awareness of risk of this complication when evaluating a neonate with OI.
\end{abstract}

\section{Publication History:}

Received: November 13, 2014

Accepted: January 10, 2015

Published: January 12, 2015

\section{Keywords:}

Collagen type I, Intracranial hemorrhage, Neonate

\section{Abbreviations: \\ Osteogenesis Imperfecta: OI \\ Collagen type I alpha 1: COL1A1 collagen type I alpha 2: COL1A2}

\section{Introduction}

Osteogenesis imperfecta (OI) is an autosomal dominant genetic disorder characterized by bone fragility with susceptibility to fracture. OI is characterized by blue sclera, dentinogenesis imperfecta, hyperlaxity of ligaments and skin, hearing impairment, and presence of wormian bones on skull radiographs [1]. Severity of symptoms varies widely, ranging from intrauterine fractures and perinatal lethality to very mild forms without fractures [2]. The majority of individuals with OI have dominantly inherited mutations in either COL1A1 or COL1A2, which encode the a chains of type I collagen [3]. Type I collagen known to be widely distributed throughout the body, including the perivascular spaces, the cardiac valves and the major extracellular components of the cerebral arterial wall [4]. Aortic dissections, carotid cavernous fistulas, cervical artery dissections, and ulnar artery aneurysms has been reported rarely to be associated with OI in the literature [5]. However, little is known about the effect on the vasculature of central nervous system. Thus, we report a case of neonate with OI type III who presented intracranial hemorrhage with a novel mutation in COL1A2.

A male neonate was transferred to our neonatal intensive care unit with a chief complain of a craniotabes found at the day of birth. No specific antenatal problems were found in the prenatal ultrasonography. The baby weighted 2,990 gram and was born at 40 weeks gestation after Cesarean section. His length was $50 \mathrm{~cm}$ and the head circumference was $34 \mathrm{~cm}$ (both at 75th percentiles). Apgar scores were eight and nine at one and five minutes, respectively. Physical examination at the time of birth showed a wide anterior fontanel $(5 \times 5 \mathrm{~cm})$. The eyes showed no proptosis and the sclerae were white. In a family history, his father had full dentures in his forties and his sclerae were blue colored. No abnormal finding was found in a neurologic examination. A radiologic image of the patient revealed irregular osteolytic cortical thinning of skull, bowing deformity of left femur and old fracture of fibular shaft (Figure 1a \& 1b). The thyroid function test and the serum levels of calcium, phosphorus, and alkaline phosphatase were within normal limits.

Echocardiography was performed as a cardiac murmur was noted. It revealed a mild pulmonary stenosis and abdominal sonography showed no abnormality. No hearing disturbance was reported by brain auditory evoked potential test. As the brain sonography suggested a cerebellar tonsillar herniation, magnetic resonance imaging (MRI) was performed. It showed no cerebellar herniation, but a subcortical and gyri form high signal intensity in both parietal lobes (dominantly left sided) was noted on coronal T1 weighted images (Figure 1c). Along the pial surface of brain parenchyma, low signal intensity with tubular shapes was noted on T2 weighted image suggesting a possibility of a vasculopathy (Figure 1d) and high signal intensity on T1 weighted image as well (figure not shown). These findings suggested acute to early subacute hemorrhage or hematoma.

Based on clinical and radiologic findings, the diagnosis of OI type III was made in reference to the NIH Osteoporosis and Related Bone Diseases guideline. Genetic testing revealed a novel mutation, heterozygous c.1036-2A >C (IVS19) in COL1A2 gene (Figure 2). His father was tested for the genetic study. He was found to carry the same mutation and diagnosed with dentinogenesis imperfecta.

\section{Methods}

Genomic DNA was isolated and a direct sequencing was performed for all 51 exons of the COL1A1 and all 52 exons of the COL1A2 using the BigDye Terminator Cycle sequencing Ready Reaction Kit version 3.1 and 3130 Genetic Analyzer (Applied Biosystems).

\section{Discussion}

Mutations in one of COL1A1 and COL1A2 are found in $90 \%$ of OI patients. The main types of OI include the milder types I and IV, the severe and fatal infantile type II, and the type III trait that is

"Corresponding Author: Dr. Won-Ho Hahn, Department of Pediatrics, Soon Chun Hyang University Seoul Hospital, 59, Daesagwan-ro, Yongsan-gu, Seoul, Republic of Korea, 140-887, Tel: +82-2-709-9147, Fax: +82-2-794-5471; E-mail: dr.hahn.md@gmail.com

Citation: Kim SH, Hahn WH (2015) A Case of Intracranial Hemorrhage in a Neonate with Osteogenesis Imperfecta Type III with a Novel Mutation in COL1A2. Int J Pediatr Neonat Care 1: 101. doi: http://dx.doi.org/10.15344/24552364/2015/101

Copyright: () $2015 \mathrm{Kim}$ et al. This is an open-access article distributed under the terms of the Creative Commons Attribution License, which permits unrestricted use, distribution, and reproduction in any medium, provided the original author and source are credited. 
Citation: Kim SH, Hahn WH (2015) A Case of Intracranial Hemorrhage in a Neonate with Osteogenesis Imperfecta Type III with a Novel Mutation in COL1A2. Int J Pediatr Neonat Care 1: 101. doi: http://dx.doi.org/10.15344/2455-2364/2015/101

Page 2 of 2

commonly associated with multiple bone fractures [6]. Collagen or genetic testing can be performed for further confirmation of the clinical diagnosis, but up to $15 \%$ of mildly affected individuals might still have negative findings [7].
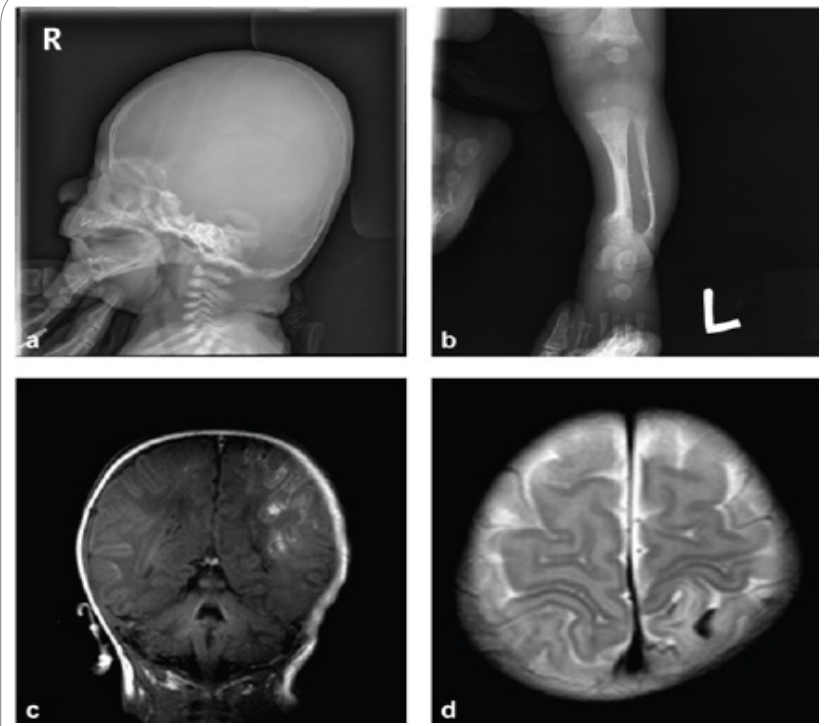

Figure 1: Radiographic findings of the newborn patient with osteogenesis imperfecta. (a) Osteolytic cortical thinning with decreased bone density of skull and flattening of skull base is noted on plain radiograph. (b) The plain radiograph of left knee shows bowing deformity of left tibiofibular shafts and fracture with callus formation of left fibula. Also, there is irregularity and spraying in metaphyseal ends of femur and tibiofibula. (c) In the brain magnetic resonance imaging scan, T1 weighted image shows gyri-form high signal intensity in both parietal lobe(left>right) with some high signal intensity foci in the sulci or subcortical white matter. (d) On T2 weighted image, periventricular and subcortical increased signal intensity on both parietal lobes (left > right) is noted. Additionally, there are multifocal parenchymal and gyral fluid collections with dark signal foci, mainly in left parietal lobe, indicating hemorrhage. This patient also had subdural hemorrhage in posterior fossa (not shown).

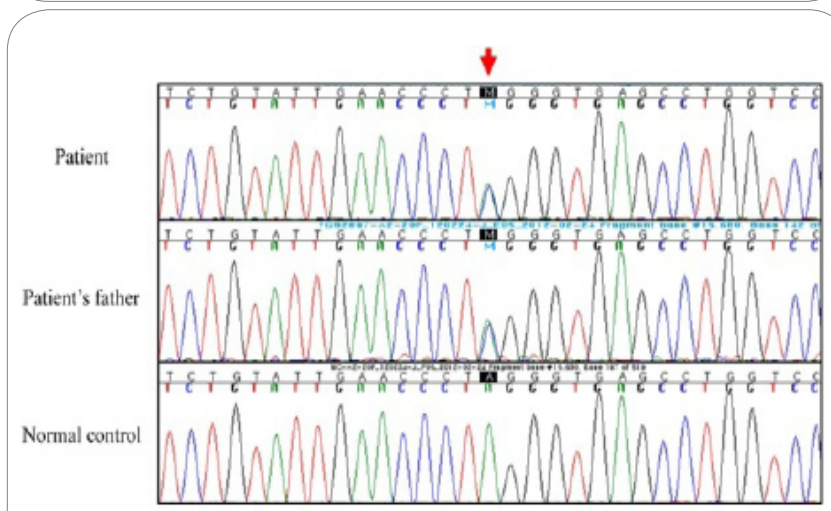

Figure 2: Mutation analysis of the COL1A2 gene. Electropherograms of the affected patient, the father and a control are shown. The arrow marks the heterozygous c.1036-2A>C, splicing variant at IVS19 of COL1A2.

Because deficient production of collagen I lead to an increased vascular fragility, decreased platelet retention and a deficient collageninduced platelet aggregation, there is an increased risk of bleeding even with only minor trauma. It might be possible that the collagen defect causes friable tissues, deficient platelet aggregation, and inadequate small vessel constriction [8]. Intracranial arterial stenosis, arterial rupture of moya-moya-like abnormality, subarachnoid hemorrhage, carotid cavernous fistula and vertebral artery fenestration may occur as a result of impaired bone calcification, vascular fragility, and friction between multiple bone fragments of the skull [9]. Intracranial hemorrhage associated with OI has been reported in a very small number of children [10].

To our knowledge, this is the first report of intracranial hemorrhage in a neonate with osteogenesis imperfecta type III. In the present case, the MRI finding revealed hemorrhage or early and subacute hematoma. Moreover, a novel base mutation (c.1036-2A>C) was detected at COL1A2 gene locus. However, the significance of this mutation is not clear including whether this mutation is responsible especially to the intracranial hemorrhage. As results from the clinical studies of the cardiovascular and neurovascular areas mentioned previously, the MRI findings in the present case suggest the possibility that the vascular fragility caused by collagen abnormality might affect the cerebral vasculature in patients with OI.

In summary, we report a rare case of OI with an unusual neurovascular complication and a novel mutation of COL1A2 gene. This case highlights the new insight into a rare but potentially critical neurovascular complication of OI neonates. Thus, we suggest keeping in mind the possibility of intracranial hemorrhage or other neurovascular lesions when evaluating a neonate with OI.

\section{Competing Interests}

The authors declare that they have no competing interests.

\section{Author Contributions}

Both the authors substantially contributed to the study design and drafting of the manuscript.

\section{References}

1. Rauch F, Glorieux FH (2004) Osteogenesis imperfecta. Lancet 363: 1377 1385.

2. Shapiro JR, Stover ML, Burn VE, McKinstry MB, Burshell AL, et al. ( 1992) An osteopenic nonfracture syndrome with features of mild osteogenesis imperfecta associated with the substitution of a cysteine for glycine at triple helix position 43 in the pro alpha $1(\mathrm{I})$ chain of type I collagen. J Clin Invest 89: $567-573$.

3. Germain-Lee EL (2011) A new culprit in osteogenesis imperfecta. J Bone Miner Res 26: 2795-2797.

4. Wheeler VR, Cooley NR Jr, Blackburn WR (1988) Cardiovascular pathology in osteogenesis imperfecta type IIA with a review of the literature. Pediatr Pathol 8: 55-64.

5. Lachman RS, Tiller GE, Graham JM Jr, Rimoin DL (1992) Collagen, genes and the skeletal dysplasias on the edge of a new era: a review and update. Eur J Radiol 14: 1-10.

6. Sillence DO, Senn A, Danks DM (1979) Genetic heterogeneity in osteogenesis imperfecta. J Med Genet 16: 101-116.

7. Petruzzellis M, De Blasi R, Lucivero V, Sancilio M, Prontera M, et al. (2007) Cerebral aneurysms in a patient with osteogenesis imperfecta and exon 28 polymorphism of COL1A2. AJNR Am J Neuroradiol 28: 397-398.

8. Sillence DO (1994) Craniocervical abnormalities in osteogenesis imperfecta: genetic and molecular correlation. Pediatr Radiol 24: 427-430.

9. Albayram S, Kizilkilic O, Yilmaz H, Tuysuz B, Kocer N, et al. (2003) Abnormalities in the cerebral arterial system in osteogenesis imperfecta. AJNR Am J Neuroradiol 24: 748-750.

10. Faqeih E, Roughley P, Glorieux FH, Rauch F (2009) Osteogenesis imperfecta type III with intracranial hemorrhage and brachydactyly associated with mutations in exon 49 of COL1A2. Am J Med Genet A 149A: 461-465. 